\title{
Transcriptomic analysis provides insights into foliar zinc application induced up-regulation in 2-acetyl-1-pyrroline and related transcriptional regulatory mechanism in fragrant rice
}

Gegen Bao ${ }^{1,2,3}$, Umair Ashraf ${ }^{4}$, Xiaorong Wan², Qi Zhou², Shengyu $\mathrm{Li}^{2}$, Chunling Wang ${ }^{5}$, Longxin $\mathrm{He}^{1,3}$, Xiangru Tang ${ }^{1,3^{*}}$

*Corresponding author: Tel (Fax): 020-85280204-618. E-mail: tangxr@ @scau.edu.cn

${ }^{1}$ Department of Crop Science and Technology, College of Agriculture, South China Agricultural University, Guangzhou 510642, PR China

${ }^{2}$ Guangzhou Key Laboratory for Research and Development of Crop Germplasm Resources, Zhongkai University of Agriculture and Engineering, Guangzhou 510225, PR China

${ }^{3}$ Scientific Observing and Experimental Station of Crop Cultivation in South China, Ministry of Agriculture, Guangzhou 510642, PR China

${ }^{4}$ Department of Botany, Division of Science and Technology, University of Education, Lahore, 54770, Punjab, Pakistan

${ }^{5}$ Guangdong Microbial Culture Collection Center (GDMCC), Guangdong Institute of Microbiology, Guangdong Academy of Sciences, Guangzhou 510070, Guangdong, PR China 


\section{Supporting information}

\section{Supplementary table legends:}

Table S1 Properties of the soil characteristics.

Table S2 Primer sequences of genes.

Table S3 Effects of foliar spray $\mathrm{Zn}$ on the 2AP, precursors, intermediates and the enzymes in leaves and grains of fragrant rice.

Marking the same letters means $\mathrm{P} \geq 0.05$ (LSD), there is no significant difference, the difference between different letters means $\mathrm{P}<0.05$ (LSD), and the difference is significant. C, Cultivar; T, Treatment; 7d AHS L, 7d after heading stage in leaves; 15 d AHS L, 15d after heading stage in leaves; $25 \mathrm{~d}$ AHS L, 25d after heading stage in leaves; MS G, Maturity stage in grains.

Table S4 Statistics of raw sequencing data results.

S, Sample; RR, Raw Reads; HQCRN, HQ Clean Reads Num; TR, Total Reads; MR, Mapping Ratio; UMR, Unique Mapped Reads; MMR, Multiple Mapped Reads.

Table S5 Output statistics of sequencing.

RR, Raw Reads; HQCRN, HQ Clean Reads Num.

Table S6 Gene quantity statistics.

Table S7 Go enrichment analysis.

Table S8 KEGG pathways.

SN, Sample Number; BN, Background Number. 
Table S1 Properties of the soil characteristics.

\begin{tabular}{ll}
\hline Properties & Contents \\
\hline organic matter & $23.34 \mathrm{~g} \mathrm{~kg}^{-1}$ \\
total nitrogen & $1.139 \mathrm{~g} \mathrm{~kg}^{-1}$ \\
total phosphorus & $1.136 \mathrm{~g} \mathrm{~kg}^{-1}$ \\
total potassium & $24.41 \mathrm{~g} \mathrm{~kg}^{-1}$ \\
available phosphate & $127.03 \mathrm{mg} \mathrm{kg}^{-1}$ \\
akali-hydrolyzale nitrogen & $114.27 \mathrm{mg} \mathrm{kg}^{-1}$ \\
available potassium & $61.34 \mathrm{mg} \mathrm{kg}^{-1}$ \\
pH & 6.14 \\
\hline
\end{tabular}

Table S1 Primer sequences of genes

\begin{tabular}{|c|c|}
\hline Gene name & Primer sequences \\
\hline \multirow[t]{2}{*}{ MH01g0365300 } & F 5'-AGTGGAAGCATTCGCAGACT-3' \\
\hline & R 5'-GAGATACCGGCACAAGTATC-3' \\
\hline \multirow[t]{2}{*}{ MH01g0377000 } & F 5'-CGTCGCTGACGGCAAGA-3' \\
\hline & R 5'-TCGATGGCACCATGTTTGGC-3' \\
\hline \multirow[t]{2}{*}{ MH02g0098600 } & F 5'-CTACAAGACCAACTGGATC-3' \\
\hline & R 5'-CTCGACGAACGTGACAGT-3' \\
\hline \multirow[t]{2}{*}{ MH02g0520200 } & F 5'-GTCCTTGTCAATGGACTGCT-3' \\
\hline & R 5'-CTTCTGCTTTGGCTTCCTTC-3' \\
\hline \multirow[t]{2}{*}{ MH02g0648800 } & F 5'-CACCAACCGCATCAAGTG-3' \\
\hline & R 5'-CGTTCACGGTGACGACGCTG-3' \\
\hline \multirow[t]{2}{*}{ MH02g0668200 } & F 5'-GTTGACTCGGACGCCGTC-3' \\
\hline & R 5'-GCCGATCTCCACCGCCGA-3' \\
\hline \multirow[t]{2}{*}{ MH03g0112900 } & F 5'-GTTGATCAGTACCACCGTTTC-3' \\
\hline & R 5'-GTACAAGGGCTGGCTTGA-3' \\
\hline \multirow[t]{2}{*}{ MH03g0191200 } & F 5'-ACTACCGGGAGACGTGCGAGAC-3' \\
\hline & R 5'-CGACCTCAAGAACGACCCGCG-3' \\
\hline \multirow[t]{2}{*}{ MH01g0314200 } & F 5'-GACTACGTGAGGCTCCACAAC-3' \\
\hline & R 5'-GAACCTCTTCTGGGGTTCGG-3' \\
\hline \multirow[t]{2}{*}{ MH01g0512100 } & F 5'-GAGATCCACGTCCGGATACGGGC-3' \\
\hline & R 5'-CGGGAGGTCCTCGCCGGCGA-3' \\
\hline \multirow[t]{2}{*}{ MH01g0793100 } & F 5'-GCTGCTCAAGAACTCGTCCTACC-3' \\
\hline & R 5'-CATGCCTGGCATGACAGGGT-3' \\
\hline \multirow[t]{2}{*}{ MH03g0082950 } & F 5'-ACGACGCTGAGCCTGATGCC-3' \\
\hline & R 5'-GACCATCTTCTACGGTGGGAG-3' \\
\hline \multirow[t]{2}{*}{ MH05g0044700 } & F 5'-ACGACGACGTGCCGTGGTTG-3' \\
\hline & R 5'-AGCCGCCGCCAAGCAGGCTC-3' \\
\hline \multirow[t]{2}{*}{ PRODH } & F 5'-TCATCAGACGAGCAGAGGAGAACAGG-3' \\
\hline & R 5'-CCCAGCATTGCAGCCTTGAACC-3' \\
\hline P5CS1 & F 5'-TTTTGAGTCCCGACCTG-3' \\
\hline
\end{tabular}




\begin{tabular}{lll} 
& R & 5'-TTCACCAACATTACGAGGA-3' \\
P5CS2 & F & 5'-GAGGTTGGCATAAGCACAG-3' \\
& R & 5'-CTCCCTTGTCGCCGTTC-3' \\
OAT & F & 5'-GCCCTTGGTGCTGGAGTA-3' \\
& R & 5'-AGCCCTTTCAACGAGACCTT-3' \\
DAO4 & F & 5'-TGGCAAGATAGAAGCAGAAGT-3' \\
& R & 5'-GTCCATACGGGCAACAAA-3' \\
DAO5 & F & 5'-TGGCAAGATAGAAGCAGAAGT-3' \\
& R & 5'-GTCCATACGGGCAACAAA-3' \\
BADH2 & F & 5'-GGTTGGTCTTCCTTCAGGTGTGC-3' \\
& R & 5'-CATCAACATCATCAAACACCACTAT-3' \\
\hline
\end{tabular}

Table S3 Effects of foliar spray $\mathrm{Zn}$ on the 2AP, precursors, intermediates and the enzymes in leaves and grains of fragrant rice.

\begin{tabular}{|c|c|c|c|c|c|c|}
\hline Index & $\mathrm{C}$ & $\mathrm{T}$ & 7d AHS L & 15d AHS L & $25 \mathrm{~d}$ AHS L & MS G \\
\hline \multirow{5}{*}{ proline } & & $\mathrm{Zn} 0$ & $289.17 \pm 45.9 \mathrm{a}$ & $77.06 \pm 19.53 b$ & $73.94 \pm 32.39 a$ & $165.85 \pm 37.7 b$ \\
\hline & M & $\mathrm{Zn} 1$ & $258.89 \pm 31.63 \mathrm{a}$ & $262.03 \pm 46.32 \mathrm{a}$ & $54.45 \pm 16.57 \mathrm{a}$ & $334.81 \pm 53.02 \mathrm{a}$ \\
\hline & & $\mathrm{Zn} 2$ & $273.9 \pm 38.74 \mathrm{a}$ & $384.46 \pm 73.05 \mathrm{a}$ & $40.09 \pm 9.73 \mathrm{a}$ & $378.97 \pm 31.7 \mathrm{a}$ \\
\hline & & $\mathrm{Zn} 0$ & $49.54 \pm 1.5 \mathrm{a}$ & $98.19 \pm 19.79 b$ & $81.79 \pm 28.95 \mathrm{a}$ & $293.93 \pm 56.68 b$ \\
\hline & $\mathrm{X}$ & $\mathrm{Zn} 1$ & $66.48 \pm 17.05 \mathrm{a}$ & $610.33 \pm 267.47 \mathrm{a}$ & $72.55 \pm 14.26 \mathrm{a}$ & $418.62 \pm 44.55 a$ \\
\hline \multirow{7}{*}{ P5C } & & $\mathrm{Zn} 2$ & $43.4 \pm 5.01 \mathrm{a}$ & $575.34 \pm 33.65 \mathrm{a}$ & $62.18 \pm 4.91 \mathrm{a}$ & $596.93 \pm 115.78 \mathrm{a}$ \\
\hline & & $\mathrm{Zn} 0$ & $1.62 \pm 0.18 b$ & $1.62 \pm 0.18 b$ & $1.45 \pm 0.03 \mathrm{a}$ & $0.59 \pm 0.24 b$ \\
\hline & M & $\mathrm{Zn} 1$ & $2.45 \pm 0.21 \mathrm{a}$ & $2.45 \pm 0.21 \mathrm{a}$ & $1.75 \pm 0.05 \mathrm{a}$ & $1.25 \pm 0.04 \mathrm{a}$ \\
\hline & & $\mathrm{Zn} 2$ & $2.82 \pm 0.04 a$ & $2.82 \pm 0.04 \mathrm{a}$ & $1.47 \pm 0.23 \mathrm{a}$ & $1.04 \pm 0.04 \mathrm{a}$ \\
\hline & & $\mathrm{Zn} 0$ & $1.8 \pm 0.12 b$ & $1.8 \pm 0.12 b$ & $1.24 \pm 0.02 \mathrm{a}$ & $0.93 \pm 0.07 b$ \\
\hline & $\mathrm{X}$ & $\mathrm{Zn} 1$ & $2.24 \pm 0.07 \mathrm{a}$ & $2.24 \pm 0.07 \mathrm{a}$ & $1.25 \pm 0.16 \mathrm{a}$ & $1.19 \pm 0.04 \mathrm{a}$ \\
\hline & & $\mathrm{Zn} 2$ & $2.43 \pm 0.14 \mathrm{a}$ & $2.43 \pm 0.14 \mathrm{a}$ & $1.29 \pm 0.26 \mathrm{a}$ & $1.54 \pm 0.09 \mathrm{a}$ \\
\hline \multirow{5}{*}{ 1-pyrroline } & & $\mathrm{Zn} 0$ & $2.69 \pm 0.25 b$ & $5.76 \pm 0.38 \mathrm{a}$ & $3.87 \pm 0.2 b$ & $2.18 \pm 0.14 b$ \\
\hline & M & $\mathrm{Zn} 1$ & $6.64 \pm 0.67 \mathrm{a}$ & $5.36 \pm 0.59 \mathrm{a}$ & $5.95 \pm 0.53 a$ & $2.39 \pm 0.08 \mathrm{ab}$ \\
\hline & & $\mathrm{Zn} 2$ & $5.59 \pm 0.24 a$ & $5.33 \pm 0.09 a$ & $6.31 \pm 0.94 a$ & $2.59 \pm 0.1 \mathrm{a}$ \\
\hline & & $\mathrm{Zn} 0$ & $6.03 \pm 0.86 a$ & $4 \pm 0.23 a$ & $3.76 \pm 0.12 b$ & $2.17 \pm 0.23 b$ \\
\hline & $\mathrm{X}$ & $\mathrm{Zn} 1$ & $4.58 \pm 0.34 \mathrm{a}$ & $4.39 \pm 0.39 \mathrm{a}$ & $4.45 \pm 0.39 \mathrm{ab}$ & $3.05 \pm 0.13 \mathrm{a}$ \\
\hline \multirow{7}{*}{ methylglyoxal } & & $\mathrm{Zn} 2$ & $5.1 \pm 0.24 \mathrm{a}$ & $5.04 \pm 0.12 \mathrm{a}$ & $5.02 \pm 0.23 \mathrm{a}$ & $3.24 \pm 0.35 \mathrm{a}$ \\
\hline & & $\mathrm{Zn} 0$ & $15.11 \pm 0.93 \mathrm{a}$ & $16.68 \pm 0.52 b$ & $21.45 \pm 2.16 b$ & $12.88 \pm 0.24 \mathrm{a}$ \\
\hline & M & $\mathrm{Zn} 1$ & $15.38 \pm 1.21 \mathrm{a}$ & $20.15 \pm 0.7 \mathrm{a}$ & $21.02 \pm 1.54 \mathrm{~b}$ & $15.38 \pm 1.21 \mathrm{a}$ \\
\hline & & $\mathrm{Zn} 2$ & $12.88 \pm 0.24 \mathrm{a}$ & $20.56 \pm 1.47 \mathrm{a}$ & $27.05 \pm 1.12 \mathrm{a}$ & $15.11 \pm 0.93 \mathrm{a}$ \\
\hline & & $\mathrm{Zn} 0$ & $6.58 \pm 0.61 \mathrm{c}$ & $6.58 \pm 0.61 \mathrm{c}$ & $15.04 \pm 0.67 \mathrm{a}$ & $6.58 \pm 0.61 \mathrm{c}$ \\
\hline & $\mathrm{X}$ & $\mathrm{Zn} 1$ & $10.74 \pm 0.67 b$ & $10.73 \pm 0.67 b$ & $23.45 \pm 1.23 \mathrm{a}$ & $10.74 \pm 0.67 b$ \\
\hline & & $\mathrm{Zn} 2$ & $13.31 \pm 1.05 \mathrm{a}$ & $13.31 \pm 1.05 \mathrm{a}$ & $15.35 \pm 1.03 \mathrm{a}$ & $13.31 \pm 1.05 \mathrm{a}$ \\
\hline \multirow{5}{*}{ GABA } & & $\mathrm{Zn} 0$ & $24.67 \pm 1.43 b$ & $12.79 \pm 4.39 \mathrm{a}$ & $17.21 \pm 3.19 \mathrm{a}$ & $15.3 \pm 0.58 \mathrm{a}$ \\
\hline & M & $\mathrm{Zn} 1$ & $31.64 \pm 1.28 \mathrm{a}$ & $17.51 \pm 4.81 \mathrm{a}$ & $8.91 \pm 1.62 b$ & $19.24 \pm 0.66 \mathrm{a}$ \\
\hline & & $\mathrm{Zn} 2$ & $27.58 \pm 1.66 \mathrm{ab}$ & $16.16 \pm 3.01 \mathrm{a}$ & $4.84 \pm 1.84 \mathrm{~b}$ & $20.39 \pm 0.11 \mathrm{a}$ \\
\hline & & $\mathrm{Zn} 0$ & $46.32 \pm 0 \mathrm{a}$ & $17.04 \pm 2.7 \mathrm{a}$ & $32.96 \pm 4.17 \mathrm{a}$ & $15.23 \pm 1.23 \mathrm{a}$ \\
\hline & $\mathrm{X}$ & $\mathrm{Zn} 1$ & $58.81 \pm 5.77 \mathrm{a}$ & $23.47 \pm 1.7 \mathrm{a}$ & $24.65 \pm 5.1 \mathrm{a}$ & $14.25 \pm 1.55 \mathrm{a}$ \\
\hline
\end{tabular}




\begin{tabular}{|c|c|c|c|c|c|c|}
\hline \multirow{7}{*}{ ProDH } & & $\mathrm{Zn} 2$ & $39.6 \pm 2.66 \mathrm{~b}$ & $17.51 \pm 4.81 \mathrm{a}$ & $24.26 \pm 8.55 \mathrm{a}$ & $15.55 \pm 0.98 \mathrm{a}$ \\
\hline & \multirow{3}{*}{$\mathrm{M}$} & $\mathrm{Zn} 0$ & $50.17 \pm 4.69 b$ & $50.17 \pm 4.69 b$ & $80.85 \pm 2.76 a$ & $43.04 \pm 1.19 b$ \\
\hline & & $\mathrm{Zn} 1$ & $69.08 \pm 2.22 \mathrm{a}$ & $69.08 \pm 2.22 \mathrm{a}$ & $86.71 \pm 1.43 \mathrm{a}$ & $57.47 \pm 3.18 \mathrm{a}$ \\
\hline & & $\mathrm{Zn} 2$ & $64.82 \pm 2.14 \mathrm{a}$ & $64.82 \pm 2.14 \mathrm{a}$ & $69.58 \pm 3.95 b$ & $56.74 \pm 3.05 \mathrm{a}$ \\
\hline & \multirow{3}{*}{$\mathrm{X}$} & $\mathrm{Zn} 0$ & $82.3 \pm 4.73 b$ & $26.44 \pm 1.05 b$ & $45.7 \pm 5.9 \mathrm{a}$ & $74.8 \pm 4.07 \mathrm{~b}$ \\
\hline & & $\mathrm{Zn} 1$ & $88.12 \pm 7.91 \mathrm{ab}$ & $61.31 \pm 3.22 a$ & $44.74 \pm 7.4 \mathrm{a}$ & $96.26 \pm 2.85 \mathrm{a}$ \\
\hline & & $\mathrm{Zn} 2$ & $106.08 \pm 3.55 \mathrm{a}$ & $64.93 \pm 4.46 \mathrm{a}$ & $44.76 \pm 3.01 \mathrm{a}$ & $87.8 \pm 3.72 \mathrm{a}$ \\
\hline \multirow{5}{*}{ P5CS } & \multirow{3}{*}{$\mathrm{M}$} & $\mathrm{Zn} 0$ & $15.79 \pm 3.45 \mathrm{a}$ & $3.37 \pm 0.22 b$ & $3.89 \pm 0.1 \mathrm{a}$ & $0.79 \pm 0.09 a$ \\
\hline & & $\mathrm{Zn} 1$ & $19.77 \pm 1.78 \mathrm{a}$ & $3.57 \pm 0.51 \mathrm{~b}$ & $3.23 \pm 0.24 b$ & $1.09 \pm 0.24 \mathrm{a}$ \\
\hline & & $\mathrm{Zn} 2$ & $19.32 \pm 1.64 \mathrm{a}$ & $8.31 \pm 2.55 \mathrm{a}$ & $3.6 \pm 0.18 \mathrm{ab}$ & $0.93 \pm 0.13 a$ \\
\hline & \multirow{3}{*}{$X$} & $\mathrm{Zn} 0$ & $42.5 \pm 2.45 \mathrm{a}$ & $2.27 \pm 1.2 \mathrm{c}$ & $5.04 \pm 1.02 \mathrm{a}$ & $0.63 \pm 0.06 \mathrm{c}$ \\
\hline & & $\mathrm{Zn} 1$ & $50.93 \pm 1.96 \mathrm{a}$ & $6.69 \pm 0.1 b$ & $7.47 \pm 1.08 \mathrm{a}$ & $2.02 \pm 0.34 \mathrm{~b}$ \\
\hline \multirow{7}{*}{ DAO } & & $\mathrm{Zn} 2$ & $40.04 \pm 6.06 \mathrm{a}$ & $8.57 \pm 1.2 \mathrm{a}$ & $7.62 \pm 1.43 \mathrm{a}$ & $3.47 \pm 0.75 \mathrm{a}$ \\
\hline & \multirow{3}{*}{$\mathrm{M}$} & $\mathrm{Zn} 0$ & $0.53 \pm 0.14 b$ & $0.81 \pm 0.12 b$ & $1.57 \pm 0.13 \mathrm{a}$ & $0.59 \pm 0.11 b$ \\
\hline & & $\mathrm{Zn} 1$ & $1.59 \pm 0.28 \mathrm{a}$ & $1.95 \pm 0.14 \mathrm{a}$ & $3.47 \pm 0.83 \mathrm{a}$ & $0.8 \pm 0.17 b$ \\
\hline & & $\mathrm{Zn} 2$ & $2.98 \pm 0.61 \mathrm{a}$ & $1.48 \pm 0.2 \mathrm{a}$ & $2.85 \pm 0.88 \mathrm{a}$ & $1.06 \pm 0.22 \mathrm{a}$ \\
\hline & \multirow{3}{*}{$\mathrm{X}$} & $\mathrm{Zn} 0$ & $0.81 \pm 0.1 \mathrm{a}$ & $0.62 \pm 0.25 b$ & $1.69 \pm 0.49 \mathrm{a}$ & $1.52 \pm 0.32 b$ \\
\hline & & $\mathrm{Zn} 1$ & $1.3 \pm 0.23 \mathrm{a}$ & $0.99 \pm 0.13 \mathrm{a}$ & $0.69 \pm 0.14 b$ & $2.75 \pm 0.52 \mathrm{a}$ \\
\hline & & $\mathrm{Zn} 2$ & $1.19 \pm 0.25 \mathrm{a}$ & $1.53 \pm 0.31 \mathrm{a}$ & $1.16 \pm 0.24 \mathrm{ab}$ & $2.01 \pm 0.44 \mathrm{ab}$ \\
\hline \multirow{5}{*}{ OAT } & \multirow{3}{*}{$\mathrm{M}$} & $\mathrm{Zn} 0$ & $1.14 \pm 0.05 \mathrm{a}$ & $1.99 \pm 0.11 \mathrm{~b}$ & $3.3 \pm 0.18 \mathrm{a}$ & $0.79 \pm 0.09 \mathrm{a}$ \\
\hline & & $\mathrm{Zn} 1$ & $1.17 \pm 0.06 \mathrm{a}$ & $2.84 \pm 0.12 b$ & $3.26 \pm 0.15 \mathrm{a}$ & $1.09 \pm 0.24 \mathrm{a}$ \\
\hline & & $\mathrm{Zn} 2$ & $1.11 \pm 0.04 \mathrm{a}$ & $3.2 \pm 0.07 \mathrm{a}$ & $3.36 \pm 0.06 \mathrm{a}$ & $0.93 \pm 0.13 \mathrm{a}$ \\
\hline & \multirow{3}{*}{$\mathrm{X}$} & $\mathrm{Zn} 0$ & $1.48 \pm 0.1 \mathrm{~b}$ & $1.48 \pm 0.1 \mathrm{~b}$ & $1.43 \pm 0.18 b$ & $2.02 \pm 0.34 \mathrm{~b}$ \\
\hline & & $\mathrm{Zn} 1$ & $1.55 \pm 0.04 \mathrm{ab}$ & $1.55 \pm 0.04 \mathrm{a}$ & $1.9 \pm 0.07 \mathrm{a}$ & $0.63 \pm 0.06 c$ \\
\hline \multirow{7}{*}{ BADH2 } & & $\mathrm{Zn} 2$ & $1.71 \pm 0.05 \mathrm{a}$ & $1.71 \pm 0.05 \mathrm{a}$ & $2.23 \pm 0.11 \mathrm{a}$ & $3.47 \pm 0.75 \mathrm{a}$ \\
\hline & \multirow{3}{*}{$\mathrm{M}$} & $\mathrm{Zn} 0$ & $24.17 \pm 1.28 \mathrm{a}$ & $27.56 \pm 1.71 \mathrm{a}$ & $27.38 \pm 2.08 \mathrm{a}$ & $27.56 \pm 1.71 \mathrm{a}$ \\
\hline & & $\mathrm{Zn} 1$ & $36.3 \pm 5.28 \mathrm{a}$ & $35.88 \pm 2.86 \mathrm{a}$ & $32.7 \pm 2.5 \mathrm{a}$ & $35.88 \pm 2.86 \mathrm{a}$ \\
\hline & & $\mathrm{Zn} 2$ & $37.34 \pm 7.02 \mathrm{a}$ & $27.97 \pm 1.43 \mathrm{a}$ & $30.13 \pm 2.9 \mathrm{a}$ & $27.97 \pm 1.43 \mathrm{a}$ \\
\hline & \multirow{3}{*}{$\mathrm{X}$} & $\mathrm{Zn} 0$ & $19.86 \pm 3.09 \mathrm{a}$ & $19.86 \pm 3.09 \mathrm{a}$ & $74.4 \pm 5.17 \mathrm{a}$ & $19.86 \pm 3.09 \mathrm{a}$ \\
\hline & & $\mathrm{Zn} 1$ & $19.09 \pm 1.62 \mathrm{a}$ & $19.06 \pm 1.62 \mathrm{a}$ & $76.46 \pm 2.04 \mathrm{a}$ & $19.06 \pm 1.62 \mathrm{a}$ \\
\hline & & $\mathrm{Zn} 2$ & $15.47 \pm 3.83 \mathrm{a}$ & $15.47 \pm 3.38 \mathrm{a}$ & $69.43 \pm 6.3 \mathrm{a}$ & $15.47 \pm 3.38 \mathrm{a}$ \\
\hline
\end{tabular}

Marking the same letters means $\mathrm{P} \geq 0.05$ (LSD), there is no significant difference, the difference between different letters means $\mathrm{P}<0.05$ (LSD), and the difference is significant. $\mathrm{C}$, Cultivar; T, Treatment; 7d AHS L, 7d after heading stage in leaves; $15 \mathrm{~d}$ AHS L, 15d after heading stage in leaves; $25 \mathrm{~d}$ AHS L, 25d after heading stage in leaves; MS G, Maturity stage in grains. 
Table S4. Statistics of raw sequencing data results.

\begin{tabular}{ccccccl}
\hline $\mathrm{S}$ & $\mathrm{RR}$ & HQCRN & TR & MR & UMR & MMR \\
\hline $\mathrm{M}-Z n 0$ & 46015854 & 44682844 & 43550410 & $85.81 \%$ & $84.975 \%$ & $0.835 \%$ \\
$\mathrm{M}-\mathrm{Zn} 1$ & 46045839 & 44793820 & 43158474 & $85.99 \%$ & $85.2 \%$ & $0.79 \%$ \\
$\mathrm{M}-\mathrm{Zn} 2$ & 53407629 & 52014541 & 50598696 & $86.77 \%$ & $85.945 \%$ & $0.82 \%$ \\
$\mathrm{X}-\mathrm{Zn} 0$ & 47337757 & 46112596 & 44761336 & $86.49 \%$ & $85.745 \%$ & $0.75 \%$ \\
$\mathrm{X}-\mathrm{Zn} 1$ & 49868783 & 48615104 & 46802711 & $86.44 \%$ & $85.57 \%$ & $0.87 \%$ \\
$\mathrm{X}-\mathrm{Zn} 2$ & 48085325 & 46916558 & 45228420 & $86.17 \%$ & $85.39 \%$ & $0.78 \%$ \\
\hline
\end{tabular}

S, Sample; RR, Raw Reads; HQCRN, HQ Clean Reads Num; TR, Total Reads; MR, Mapping Ratio; UMR, Unique Mapped Reads; MMR, Multiple Mapped Reads.

Table S5. Output statistics of sequencing.

\begin{tabular}{cccclc}
\hline Sample & RR & HQCRN & Q20(\%) & Q30(\%) & GC(\%) \\
\hline M-Zn0 & 46015854 & 44682844 & $98.025 \%$ & $94.04 \%$ & $54.605 \%$ \\
M-Zn1 & 46045839 & 44793820 & $98.08 \%$ & $94.155 \%$ & $54.705 \%$ \\
M-Zn2 & 53407629 & 52014541 & $98.115 \%$ & $94.22 \%$ & $55.165 \%$ \\
X-Zn0 & 47337757 & 46112596 & $98.09 \%$ & $94.16 \%$ & $55.36 \%$ \\
X-Zn1 & 49868783 & 48615104 & $98.125 \%$ & $94.25 \%$ & $55 \%$ \\
X-Zn2 & 48085325 & 46916558 & $98.035 \%$ & $94.01 \%$ & $55.255 \%$ \\
\hline
\end{tabular}

RR, Raw Reads; HQCRN, HQ Clean Reads Num.

Table S6. Gene quantity statistics.

\begin{tabular}{cccc}
\hline Sample & Known Gene Num & New Gene Num & All Gene Num \\
\hline M-Zn0 & $25394(44.41 \%)$ & 352 & 25746 \\
M-Zn1 & $25367(44.36 \%)$ & 350 & 25717 \\
M-Zn2 & $25680(44.91 \%)$ & 351 & 26031 \\
X-Zn0 & $24999(43.72 \%)$ & 329 & 25328 \\
X-Zn1 & $25418(44.45 \%)$ & 329 & 25747 \\
X-Zn2 & $25310(44.26 \%)$ & 327 & 25637 \\
All Reference Genes & $31534(55.14 \%)$ & 361 & 57185 \\
\hline
\end{tabular}


Table S7. Go enrichment analysis.

\section{a Biological Process}

\section{Go Description}

cellular component organization or biogenesis cellular process

growth

metabolic process

immune system process

localization

reproduction

multicellular organismal process

multi-organism process

signaling

reproductive process

developmental process

biological regulation

response to stimulus

single-organism process

\section{b Molecular Function}

catalytic activity

molecular transducer activity

transporter activity

nucleic acid binding transcription factor activity

structural molecule activity

binding

antioxidant activity

\section{c Cellular Component}

membrane part

membrane

extracellular region

cell part

cell

organelle

organelle part

macromolecular complex

\begin{tabular}{|c|c|c|c|}
\hline 1 & 17 & 2 & 8 \\
\hline 29 & 96 & 29 & 58 \\
\hline 27 & 1 & & 1 \\
\hline & 111 & 32 & 67 \\
\hline 5 & 1 & & 10 \\
\hline 1 & 20 & 7 & 1 \\
1 & 3 & 1 & 2 \\
\hline & 4 & 2 & 1 \\
\hline 1 & 1 & & \\
1 & 3 & & 2 \\
\hline 2 & 2 & 1 & 14 \\
\hline 1 & 4 & 2 & 4 \\
\hline 18 & 21 & 5 & 32 \\
\hline
\end{tabular}

\begin{tabular}{|c|c|c|c|}
\hline 31 & 120 & 38 & 70 \\
\hline 5 & 8 & 1 & 6 \\
\hline 1 & 2 & 7 & 1 \\
\hline 28 & 1 & & 50 \\
\hline
\end{tabular}

\begin{tabular}{|c|c|c|c|}
\hline 11 & 22 & 12 & 12 \\
13 & 25 & 15 & 14 \\
16 & 55 & 16 & 31 \\
16 & 55 & 13 & 31 \\
15 & 42 & 12 & 25 \\
4 & 8 & 2 & 6 \\
4 & 7 & 2 & 4 \\
\hline
\end{tabular}


Table S8. KEGG pathways.

\begin{tabular}{lcccc}
\hline Pathway & SN & BN & P-Value & Comparison \\
\hline Plant-pathogen interaction & 5 & 276 & 0.020057 & M-Zn0-vs-M-Zn1 \\
SNARE interactions in vesicular transport & 2 & 41 & 0.023185 & M-Zn0-vs-M-Zn1 \\
Mismatch repair & 2 & 61 & 0.048171 & M-Zn0-vs-M-Zn1 \\
Starch and sucrose metabolism & 17 & 238 & 0.000004 & M-Zn0-vs-M-Zn2 \\
Pentose and glucuronate interconversions & 10 & 81 & 0.000004 & M-Zn0-vs-M-Zn2 \\
Plant-pathogen interaction & 16 & 276 & 0.000094 & M-Zn0-vs-M-Zn2 \\
Nitrogen metabolism & 5 & 40 & 0.001063 & M-Zn0-vs-M-Zn2 \\
Photosynthesis - antenna proteins & 3 & 16 & 0.003526 & M-Zn0-vs-M-Zn2 \\
Cyanoamino acid metabolism & 5 & 57 & 0.005181 & M-Zn0-vs-M-Zn2 \\
Phenylpropanoid biosynthesis & 11 & 247 & 0.009468 & M-Zn0-vs-M-Zn2 \\
Plant hormone signal transduction & 11 & 281 & 0.022912 & M-Zn0-vs-M-Zn2 \\
Lysine degradation & 3 & 38 & 0.038792 & M-Zn0-vs-M-Zn2 \\
Photosynthesis & 4 & 67 & 0.043315 & M-Zn0-vs-M-Zn2 \\
Flavone and flavonol biosynthesis & 1 & 4 & 0.025738 & X-Zn0-vs-X-Zn1 \\
Fatty acid elongation & 2 & 47 & 0.036958 & X-Zn0-vs-X-Zn1 \\
Diterpenoid biosynthesis & 2 & 49 & 0.039883 & X-Zn0-vs-X-Zn1 \\
Starch and sucrose metabolism & 11 & 238 & 0.000131 & X-Zn0-vs-X-Zn2 \\
Pentose and glucuronate interconversions & 6 & 81 & 0.000436 & X-Zn0-vs-X-Zn2 \\
Diterpenoid biosynthesis & 3 & 49 & 0.021885 & X-Zn0-vs-X-Zn2 \\
Zeatin biosynthesis & 2 & 24 & 0.034525 & X-Zn0-vs-X-Zn2 \\
\hline
\end{tabular}

SN, Sample Number; BN, Background Number. 